\title{
Fast Synaptic Currents in Drosophila Mushroom Body Kenyon Cells Are Mediated by $\alpha$-Bungarotoxin-Sensitive Nicotinic Acetylcholine Receptors and Picrotoxin-Sensitive GABA Receptors
}

\author{
Hailing Su and Diane K. O'Dowd \\ Department of Anatomy and Neurobiology and Department of Developmental and Cell Biology, University of California Irvine, Irvine, California \\ 92697-1280
}

The mushroom bodies, bilaterally symmetric regions in the insect brain, play a critical role in olfactory associative learning. Genetic studies in Drosophila suggest that plasticity underlying acquisition and storage of memory occurs at synapses on the dendrites of mushroom body Kenyon cells (Dubnau et al., 2001). Additional exploration of the mechanisms governing synaptic plasticity contributing to these aspects of olfactory associative learning requires identification of the receptors that mediate fast synaptic transmission in Kenyon cells. To this end, we developed a culture system that supports the formation of excitatory and inhibitory synaptic connections between neurons harvested from the central brain region of late-stage Drosophila pupae. Mushroom body Kenyon cells are identified as small-diameter, green fluorescent protein-positive (GFP + ) neurons in cultures from 0K107-GAL4;UAS-GFP pupae. In GFP + Kenyon cells, fast EPSCs are mediated by $\alpha$-bungarotoxin-sensitive nicotinic acetylcholine receptors (nAChRs). The miniature EPSCs have rapid rise and decay kinetics and a broad, positively skewed amplitude distribution. Fast IPSCs are mediated by picrotoxin-sensitive chloride conducting GABA receptors. The miniature IPSCs also have a rapid rate of rise and decay and a broad amplitude distribution. The vast majority of spontaneous synaptic currents in the cultured Kenyon cells are mediated by $\alpha$-bungarotoxin-sensitive nAChRs or picrotoxinsensitive GABA receptors. Therefore, these receptors are also likely to mediate synaptic transmission in Kenyon cells in vivo and to contribute to plasticity during olfactory associative learning.

Key words: Drosophila; Kenyon cells; mushroom bodies; synaptic transmission; mEPSCs; mIPSCs; cholinergic; GABAergic

\section{Introduction}

A combination of behavioral and genetic studies in honeybees and Drosophila have demonstrated that an anatomically distinct region of the adult insect brain, the mushroom body, plays an important role in olfactory associative learning (Heisenberg, 1998). However, the sites of synaptic plasticity that mediate the behavioral changes have been more difficult to pinpoint. Recently, reversible disruption of transmitter release in Drosophila mushroom body Kenyon cells, using a temperature-sensitive dynamin transgene, was shown to block memory retrieval but not acquisition or storage (Dubnau et al., 2001). This suggests that synapses formed on the dendrites of the Kenyon cells are sites of plasticity contributing to olfactory associative learning. Testing this hypothesis requires identification of the specific mushroom body afferents activated during olfactory learning and functional characterization of the receptors mediating synaptic activity in the Kenyon cells.

\footnotetext{
Received June 10, 2003; revised Aug. 27, 2003; accepted Aug. 29, 2003.

This work was supported by National Institutes of Health Grants NS27501 and DA14960. We thank Kevin Boswell, Betty Sicaeros, and Renna Pandhi for technical assistance with culturing and Dr. M. A. Smith for helpful comments on previous versions of this manuscript.

Correspondence should be addressed to Dr. Diane K. O'Dowd, Department of Anatomy and Neurobiology, University of California Irvine, Irvine, CA 92697-1280. E-mail: dkodowd@uci.edu.

Copyright $\odot 2003$ Society for Neuroscience $\quad 0270-6474 / 03 / 239246-08 \$ 15.00 / 0$
}

Although the neural circuits activated by foot shock, the unconditioned stimulus presented during olfactory associative conditioning, are unknown, functional processing of olfactory stimuli has been studied extensively in the insect CNS (Hansson, 2002; Theunissen, 2003). In vivo intracellular recordings in locust demonstrate that Kenyon cells receive both excitatory and inhibitory inputs in response to odors (Perez-Orive et al., 2002). Excitatory information arrives from cholinergic projection neurons (PNs) located in the antennal lobe. GABAergic lateral horn neurons, activated by odors, provide inhibitory input to the Kenyon cells. Although there are no reports of in vivo intracellular recordings from Drosophila or honeybee Kenyon cells, anatomical studies indicate that axons from antennal lobe PNs are the primary source of cholinergic input to the mushroom body neurons in these insects as well (Kreissl and Bicker, 1989; Bicker, 1999; Yusuyama et al., 2002). In addition, ultrastructural analyses have identified GABA-positive boutons on Kenyon cell dendrites in Drosophila (Yusuyama et al., 2002) and honeybees (Ganeshina and Menzel, 2001).

Despite the strong evidence that both cholinergic and GABAergic mushroom body afferents are activated during olfactory information processing, the functional properties of the receptors mediating fast synaptic transmission in Kenyon cells remain unknown. Studies in locust demonstrate that electrical 


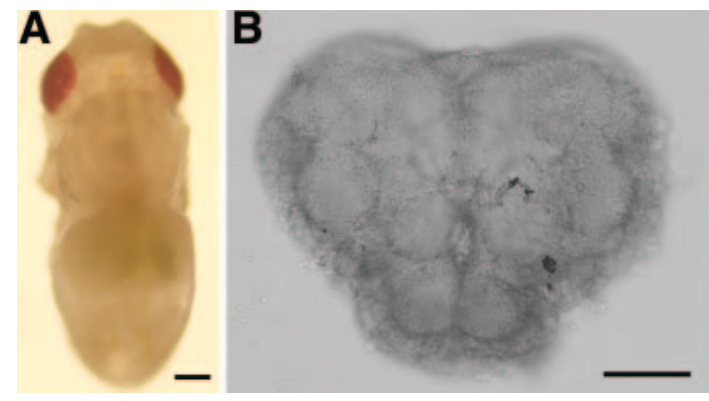

Figure 1. Central brain region of a late-stage wild-type Drosophila pupa. A, Lowmagnification photomicrograph of a Canton-S wild-type pupa identified as stage 10 (red eyes, bald; $\sim 70 \mathrm{hr}$ after pupation). Scale bar, $200 \mu \mathrm{m}$. B, Bright-field image of an intact central brain region removed from a stage 10 Canton- $S$ pupa. The tissue, slightly flattened by a glass coverslip, is oriented with the anterior face up and dorsal at the top. Scale bar, $100 \mu \mathrm{m}$.

stimulation of the antennal lobe results in complex postsynaptic potentials in Kenyon cells with both cholinergic and GABAergic components (Perez-Orive et al., 2002). However, the properties of the underlying synaptic currents have not yet been described. Although locust is the only insect in which there has been electrophysiological analysis of Kenyon cells in vivo, application of acetylcholine and GABA reveals that Kenyon cells cultured from cricket and honeybee express both nicotinic acetylcholine receptor (nAChR) and GABA receptors (Cayre et al., 1999; Goldberg et al., 1999; Deglise et al., 2002). However, no evidence of synaptic connectivity between neurons in these culture systems has precluded their use in the identification of the receptors that mediate synaptic transmission. Here we report conditions in which neurons from the central brain region of late-stage Drosophila pupae form functional synaptic connections in culture. In the Kenyon cells, identified as green fluorescent protein-positive $(\mathrm{GFP}+)$ neurons in cultures made from OK107-GAL4;UAS-GFP pupae, $\alpha$-bungarotoxin-sensitive nAChRs mediate excitatory synaptic currents, and picrotoxin-sensitive GABA receptors mediate inhibitory synaptic currents.

\section{Materials and Methods}

Fly strains. Pupae from two wild-type strains were used, Canton-S and Oregon-R. Pupae were also obtained from the mating of males from the homozygous enhancer trap line OK107-GAL4 (Connolly et al., 1996) and females homozygous for a UAS-GFP transgene (both lines obtained from Y. Zhong, Cold Spring Harbor Laboratory, Cold Spring Harbor, NY). The OK107-GAL4;UAS-GFP pupae exhibit high levels of GFP expression in the cell bodies and processes of the mushroom body Kenyon cells in late-stage pupae (see Fig. $4 A$ ). The Cha-GAL line used in the study was a $7.4 \mathrm{~kb}$ Cha-Gal4 driver line (19B) recombined with UASGFP (S65T) (Salvaterra and Kitamoto, 2001). As shown previously, cholinergic neurons in the brains of these animals are GFP+.

Primary neuronal cultures from pupal CNS. The heads were removed from animals at pupal stage $8-10$, which corresponds to $\sim 50-78 \mathrm{hr}$ after pupation (Bainbridge and Brownes, 1981). In the wild-type strains these stages were identified by the presence of yellow-red pigmentation in the eye, in the absence of significant pigmentation elsewhere in the animal (Fig. $1 A$ ). Immediately after decapitation, the heads were placed in sterile dissecting saline containing the following (in $\mathrm{mm}$ ): $126 \mathrm{NaCl}, 5.4 \mathrm{KCl}$, $0.17 \mathrm{NaH}_{2} \mathrm{PO}_{4}, 0.22 \mathrm{KH}_{2} \mathrm{PO}_{4}, 33.3$ glucose, 43.8 sucrose, and 9.9 HEPES, $\mathrm{pH}$ 7.4. The brains were removed and the optic lobes discarded. Central brain regions (Fig. $1 B$ ) from 5-15 animals were incubated in dissecting saline containing $50 \mathrm{U} / \mathrm{ml}$ papain activated by L-cysteine $(1.32 \mathrm{~mm})$ for 15 min at room temperature. Tissue was washed (1.5 ml, four times) with Drosophila-defined culture medium (DDM2) composed of Ham's F-12 DMEM (high glucose) (Irvine Scientific, Santa Ana, CA) supplemented with $1 \mathrm{mg} / \mathrm{ml}$ of sodium bicarbonate, $20 \mathrm{~mm}$ HEPES, $100 \mu \mathrm{m}$ putrescine,
$30 \mathrm{~nm}$ sodium selenite, $20 \mathrm{ng} / \mathrm{ml}$ of progesterone, $50 \mu \mathrm{g} / \mathrm{ml}$ of insulin, 100 $\mu \mathrm{g} / \mathrm{ml}$ of transferrin, and $1 \mu \mathrm{g} / \mathrm{ml}$ of 20 -hydroxyecdysone. Single brains were transferred to a $5 \mu \mathrm{l}$ drop of DDM2 culture medium placed on a concanavalin A (Con A)-laminin-coated (Kraft et al., 1998) glass coverslip in a $35 \mathrm{~mm}$ plastic dish. The tissue was mechanically dissociated and the cells were allowed to settle to the substrate for $30 \mathrm{~min}$. Dishes were flooded with $1.5 \mathrm{ml}$ of DDM2 and maintained in a $23^{\circ} \mathrm{C}$, humidified, $5 \%$ $\mathrm{CO}_{2}$ incubator. After $24 \mathrm{hr}, 0.5 \mathrm{ml}$ of a 3:1 mixture of DDM2 and conditioned neurobasal medium (cNBM) was added to each dish. cNBM (neurobasal medium containing B27 supplements; Invitrogen, Gaithersburg, MD) was conditioned for $24 \mathrm{hr}$ by non-neuronal mouse brain feeder cell cultures (Hilgenberg et al., 1999). Cultures were fed every 4-5 $\mathrm{d}$ by removing $1 \mathrm{ml}$ of media from the dish and adding $1 \mathrm{ml}$ of the 3:1 mixture of DDM2 and cNBM.

Electrophysiology. Postsynaptic currents (PSCs) were recorded with whole-cell pipettes (3-10 M $\Omega$ ) filled with internal solution containing the following (in $\mathrm{mm}$ ): $120 \mathrm{CsOH}, 120 \mathrm{D}$-gluconic acid, $0.1 \mathrm{CaCl}_{2} 2$ $\mathrm{MgCl}_{2}, 20 \mathrm{NaCl}, 1.1$ EGTA, 4 ATP, and 10 HEPES, pH 7.2. The external solution contained the following (in mM): $140 \mathrm{NaCl}, 1 \mathrm{CaCl}_{2}, 4 \mathrm{MgCl}_{2}, 3$ $\mathrm{KCl}$, and 5 HEPES, $\mathrm{pH}$ 7.2. All data shown are corrected for the $5 \mathrm{mV}$ liquid junction potential generated in these solutions. PSCs examined in the presence of TTX to block voltage-gated sodium channels were defined as miniature PSCs (mPSCs). The following drugs were bathapplied in specific experiments: TTX $(1 \mu \mathrm{M})$, D-tubocurarine (curare, 1 $\mu \mathrm{M}), \alpha$-bungarotoxin ( $\alpha$-BTX, $0.1 \mu \mathrm{M}), 6$-cyano-7-nitroquinoxaline2,3-dione (CNQX, $5 \mu \mathrm{M}$ ), D(-)-2-amino-5-phosphonopentanoic acid (APV, $50 \mu \mathrm{M}$ ), bicuculline methylchloride (BMC, 2-100 $\mu \mathrm{M}$ ), picrotoxin (PTX, 2-10 $\mu \mathrm{M}$ ). Data were acquired with a List EPC7 amplifier, a Digidata 1320A D-A converter (Axon Instruments, Foster City, CA), a Dell Dimension 4100 computer (Dell Computer Company, Round Rock, TX) , and pClamp 8 (Axon Instruments) software. Recordings were made at room temperature between 3 and $21 \mathrm{~d}$ in vitro (DIV).

Analysis of synaptic currents. Spontaneous PSCs were detected using mPSC detection software (Minianalysis Program; Synaptosoft, Decatur, GA) with a threshold criterion for individual events of $7 \mathrm{pA}$ for EPSCs and $5 \mathrm{pA}$ for IPSCs. In addition, events were accepted for kinetic analysis only if the shape was asymmetrical, with a fast rising phase and more slowly decaying falling phase. Currents were filtered at $2 \mathrm{kHz}$ and digitized at $10-20 \mathrm{kHz}$. The mean amplitude and rise time were determined from the ensemble average currents assembled from 10 or more single events in each neuron. Decay time constants were determined by fitting a single exponential distribution to the falling phase of the ensemble average current from each neuron. Frequency was determined from continuous recordings of at least $1 \mathrm{~min}$ in duration.

GABA staining and analysis. Neurons were fixed in $4 \%$ paraformaldehyde for $30 \mathrm{~min}$ at room temperature. After three washes with $\mathrm{PBS} / 4 \%$ $\mathrm{BSA}$, the neurons were treated with $0.2 \%$ Triton $\mathrm{X}-100$ for $30 \mathrm{~min}$. The primary antibody (rabbit polyclonal anti-GABA antibody; Sigma, St. Louis, MO) was used at a dilution of $1: 1000$ in a $4^{\circ} \mathrm{C}$ overnight incubation. A peroxidase-conjugated donkey anti-rabbit secondary antibody was used at a dilution of 1:50 in a $1 \mathrm{hr}$, room temperature incubation. The staining was visualized with a nickel-enhanced DAB reaction. To determine the percentage of GABA-positive neurons, one field of view on each coverslip was randomly selected, and both the number of GABA-positive neurons and the total number of neurons were counted.

\section{Results}

\section{Synaptic transmission between neurons cultured from the central brain region of late-stage pupae}

The central brain region from each individual late-stage pupa was mechanically dissociated in a $5 \mu$ ldrop of culture medium, directly on a Con A-laminin-coated coverslip. Isolated cells, some retaining axon-like projections, adhere to the substrate after dissociation (Fig. 2A). The number of cells with processes increased rapidly during the first 3 DIV. Process length and complexity continued to increase for 1-2 weeks, resulting in regions of extensive morphological contact between cells (Fig. 2 B,C). Cholinergic neurons were identified as the GFP + cells in live cultures 

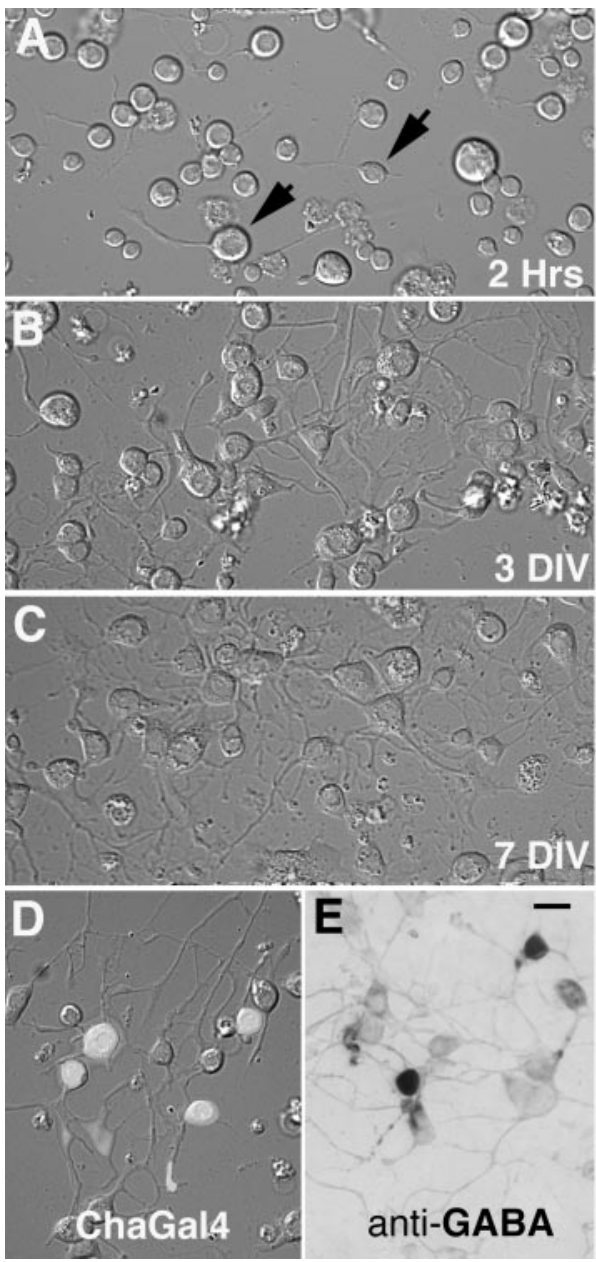

Figure 2. Cultures prepared from central brain regions of late stage pupae. $A, B, C$, Live cultures imaged with Nomarski optics. A, At $2 \mathrm{hr}$ after plating, single spherical cells adhere to the substrate. A small number of neurons retain their axon-like projections through the dissociation procedure (arrows). B, At 3 DIV a large percentage of the cells extend neuritic processes and form morphologically connected networks. C, Neurite growth and branching results in elaborate mats of interconnected processes at 7 DIV. D, GFP fluorescence identifies 4 of 11 neurons as cholinergic, in a live culture prepared from a Cha-GAL4 pupa. Fluorescent mask projected on a Nomarski image of the same field of view. Culture viewed at 3 DIV. E, Anti-GABA antibody staining identifies 2 of 10 neurons as GABAergic in a culture fixed and stained at 6 DIV. Scale bar, $10 \mu \mathrm{m}$.

prepared from Cha-GAL4 pupae, in which the regulatory DNA from the cholinergic locus is fused to GAL4 and a UAS-GFP construct (Salvaterra and Kitamoto, 2001). GFP+ cholinergic neurons (Fig. $2 D$ ) accounted for $37 \pm 2 \%$ (mean \pm SEM) of the cells extending neuritic processes in randomly selected fields of view from nine coverslips prepared in three independent platings. The GFP + cells appeared to represent a subset of the cholinergic neurons because $>80 \%$ of the neurons in fixed cultures were stained with an antibody to the vesicular acetylcholine transporter (data not shown). GABAergic neurons were identified in cultures fixed and stained with an anti-GABA antibody (Fig. 2 E). GABA-positive neurons represented $14 \pm 2 \%$ (mean \pm SEM, $n=19$ ) of the cells extending neuritic processes in a randomly selected field of view from 19 coverslips prepared in 10 independent platings. All counts were made on coverslips between 2 and $7 \mathrm{~d}$ in culture.

Initial electrophysiological data were obtained from neurons, visually selected under Hoffman illumination in the absence of fluorescence, which had processes and were in physical contact with one or more neighboring cells. Neurons chosen under these conditions were examined in cultures prepared from two wildtype strains, Canton-S and Oregon-R. In addition, cells chosen in a similar manner were also examined in two additional lines (OK107-GAL4;UAS-GFP and Cha-GAL4). In later experiments, recordings were obtained from identified cell groups in the OK107-GAL4;UAS-GFP and Cha-GAL4 cultures using fluorescent illumination.

Spontaneously occurring fast synaptic currents were observed in randomly selected neurons in cultures prepared from the brains of all four genotypes. In some of the neurons, the fast synaptic currents were inward at a holding potential of $-75 \mathrm{mV}$, outward at $0 \mathrm{mV}$, with both inward and outward synaptic currents present at $-15 \mathrm{mV}$ (Fig. $3 \mathrm{~A}$ ). This demonstrates that at least two classes of receptors, characterized by distinct reversal potentials, contribute to the spontaneous synaptic currents in the cultured neurons. The inward currents were identified as cholinergic and excitatory based on their reversible block by $1 \mu \mathrm{M}$ curare (Fig. $3 B)$ and a positive reversal potential $(8.9 \pm 1.7 \mathrm{mV}, n=9)$. Outward currents were identified as GABAergic and inhibitory based on their blockade by bath perfusion of $10 \mu \mathrm{M}$ PTX (Fig. 3B) and a reversal potential of $-37 \pm 3 \mathrm{mV}(n=4)$, close to the theoretical chloride equilibrium potential of $-45 \mathrm{mV}$. Analysis of over 100 cultures between 3 and 21 DIV revealed that the probability of observing neurons with cholinergic and/or GABAergic synaptic currents varied widely between cultures (Fig. $3 C$ ). The incidence was higher during the first 10 DIV but there were no obvious differences in cultures from the four different genotypes. These data demonstrate that pupal neurons form functional synaptic connections, mediated by $\mathrm{nAChRs}$ and GABA receptors, in dissociated cell culture.

\section{Identification of mushroom body Kenyon cells}

To assess the properties of the receptors mediating synaptic transmission specifically in Kenyon cells, the GAL4-UAS system was used to identify these neurons. Male OK107-GAL4 flies crossed to females homozygous for a chromosome containing a UAS-GFP construct result in progeny with high levels of GFP expression in the cell bodies and processes of the mushroom body Kenyon cells in late-stage pupae (Fig. 4A). GFP expression elsewhere in the central brain region is limited to several small groups of neurons. This is very similar to the pattern of expression reported previously using the same OK107-GAL4 line (Lee et al., 1999). Cultures prepared from OK107-GAL4;UAS-GFP pupal brains contained a subpopulation of neurons, $\sim 10-15 \%$ of the total, that were GFP+ (Fig. $4 B$ ). The mean number of GFP+ neurons in cultures prepared from single brains was $1303 \pm 80$ (mean $\pm \mathrm{SEM}, n=29$ cultures). This number did not change significantly between the first and second weeks in culture. The cell bodies of the GFP + neurons in these cultures were relatively small (Fig. $4 B$ ), consistent with the small soma size of this population of neurons in vivo. Using higher resistance (8-10 M $\Omega$ ) recording electrodes than in previous studies, it was possible to obtain stable whole-cell recordings from the GFP+ neurons. Consistent with the small cell body diameter of Kenyon cells, both in vivo and in vitro, the mean whole-cell capacitance of cells in which synaptic currents were evaluated was significantly smaller in GFP + Kenyon cells vs GFPnon-Kenyon cells, $3.6 \pm 0.4 \mathrm{pF}(n=38)$ and $14.0 \pm 1.1 \mathrm{pF}(n=35)$, respectively ( $p<0.001$, Student's $t$ test, unpaired).

\section{Cholinergic mEPSCs in GFP+ Kenyon cells}

The properties of the cholinergic synaptic currents in GFP+ Kenyon cells were assessed from analyses of mEPSCs recorded at $-75 \mathrm{mV}$ in the presence of TTX to block voltage-gated sodium 
A

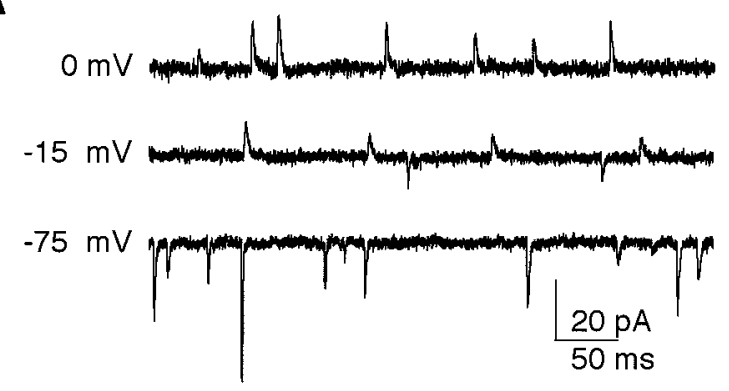

B

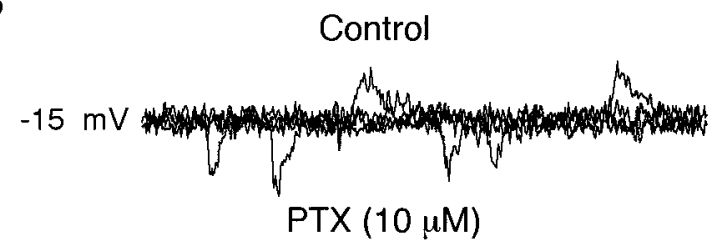

$-15 \mathrm{mV}$

Curare $(1 \mu \mathrm{M})$

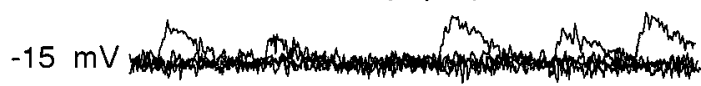

Wash

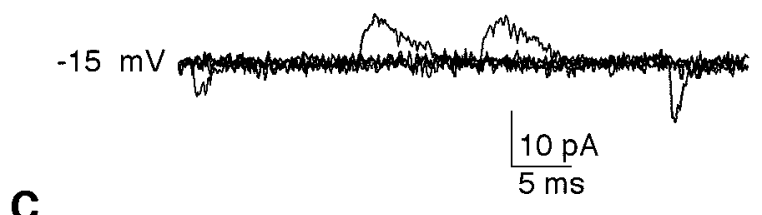

$\mathbf{C}$

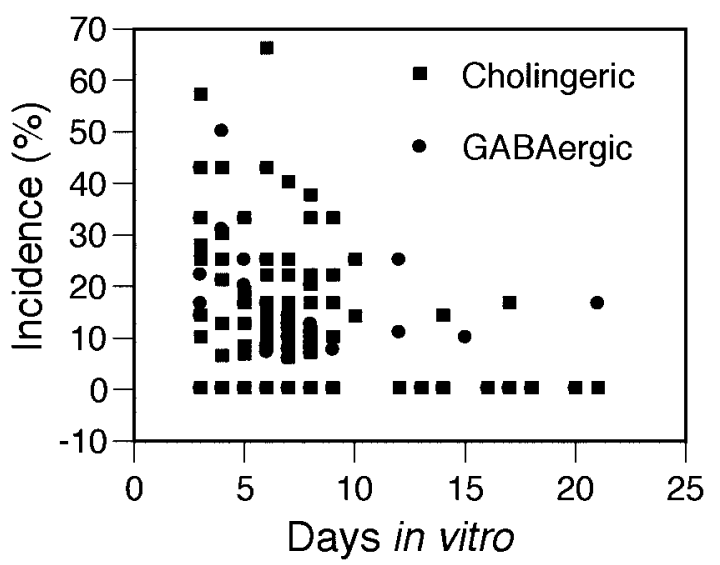

Figure 3. nAChRs and GABA receptors mediate fast synaptic currents in cultured central brain neurons. $A$, Representative traces of synaptic currents recorded from a single wild-type neuron at three different holding potentials. All currents are inward at $-75 \mathrm{mV}$ and outward at $0 \mathrm{mV}$. Both inward and outward currents are observed at $-15 \mathrm{mV}$. $B$, The outward currents recorded at $-15 \mathrm{mV}$ are reversibly blocked by bath application of PTX, and the inward currents are reversibly blocked by bath application of curare, confirming the presence of both cholinergic and GABAergic synaptic receptors in this neuron. Three traces are superimposed in each of the four records. C, Each symbol represents the incidence, calculated as the percentage of cells with synaptic currents, in which four or more stable whole-cell recordings were obtained in cultures between 3 and 21 DIV. Cultures were prepared from Canton-S, Oregon-R, Cha-GAL4, and OK107-GAL4;UAS-GFP pupae.

channels. Data were collected from cells in the presence of PTX to block the GABA receptors. As expected for nAChR-mediated currents, the mEPSCs in the Kenyon cells were completely and reversibly blocked by $1 \mu \mathrm{M}$ curare $(n=5)$ but were not blocked
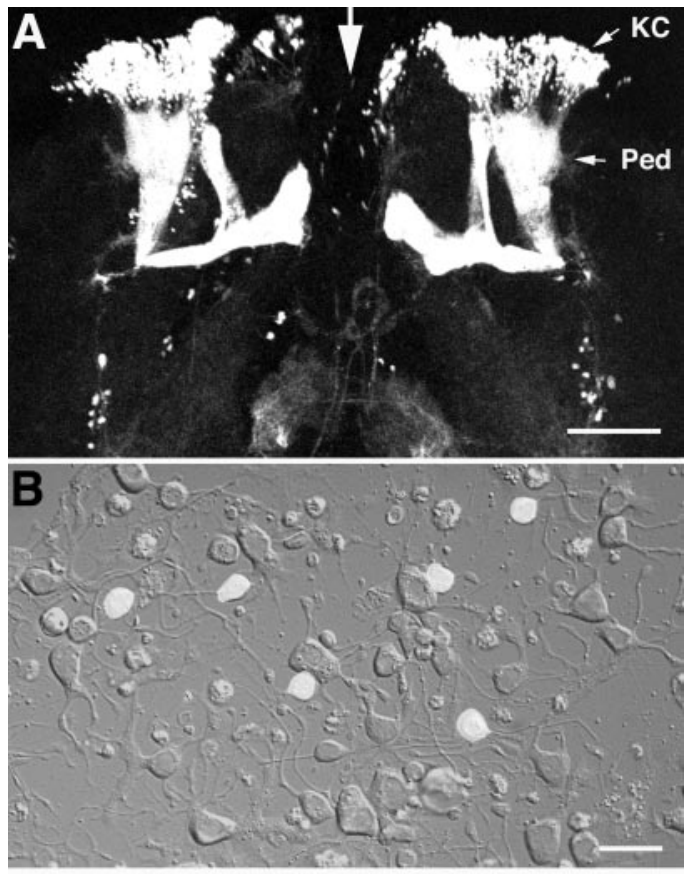

OK107-GAL4;UAS-GFP

Figure 4. GFP + neurons in the OK107-GAL4;UAS-GFP pupae identify Kenyon cells in vivo and in vitro. A, Fluorescent confocal image of a brain harvested from a stage 10, OK107-GAL4; UAS-GFP pupa. Dorsal is up and the midline of the brain is indicated by the large white arrow. KC, Mushroom body Kenyon cell region; Ped, peduncle. The brain has been flattened with a glass coverslip. Scale bar, $200 \mu \mathrm{m}$. B, Kenyon cells are identified as the small, GFP + neurons in a 4-d-old culture prepared from the central brain region of an 0K107-GAL4;UAS-GFP pupa. A fluorescent mask is projected on a Nomarski image of the same field of view. This field contains six GFP + Kenyon cells. Scale bar, $10 \mu \mathrm{m}$.
A

Control

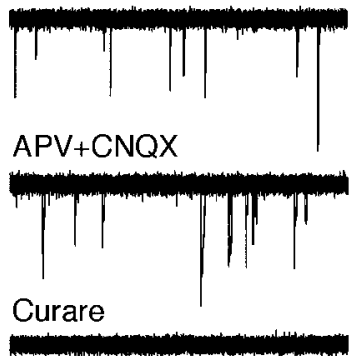

Wash

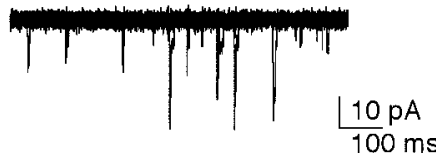

B

\section{Control}

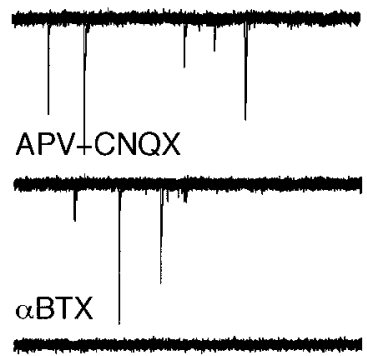

Wash

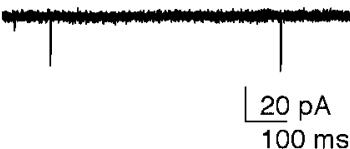

Figure 5. Pharmacological properties of cholinergic synaptic currents in GFP + Kenyon cells. $A$, Fast synaptic currents are reversibly blocked by $1 \mu \mathrm{m}$ curare but are not blocked by CNQX (5 $\mu \mathrm{M})$ and APV $(50 \mu \mathrm{m})$. Superimposed current traces (six sweeps) obtained from a single wildtype neuron, $3 \mathrm{DIV}$, at a holding potential of $-75 \mathrm{mV}$ during bath perfusion of the indicated drugs. B, Fast inward currents are blocked by $\alpha-B T X(0.1 \mu \mathrm{M})$. This blockade is partially reversible. Superimposed current traces (three sweeps) obtained from a wild-type neuron, 5 DIV, at a holding potential of $-75 \mathrm{mV}$. Cultures were prepared from 0K107-GAL4;UAS-GFP pupae.

by APV and CNQX $(n=6)$, classic glutamate receptor antagonists (Fig. 5A). In addition, bath application of $0.1 \mu \mathrm{M} \alpha$-BTX also blocked the cholinergic mEPSCs $(n=5)$ (Fig. $5 B)$. To further characterize the receptors mediating the cholinergic currents, the 
A

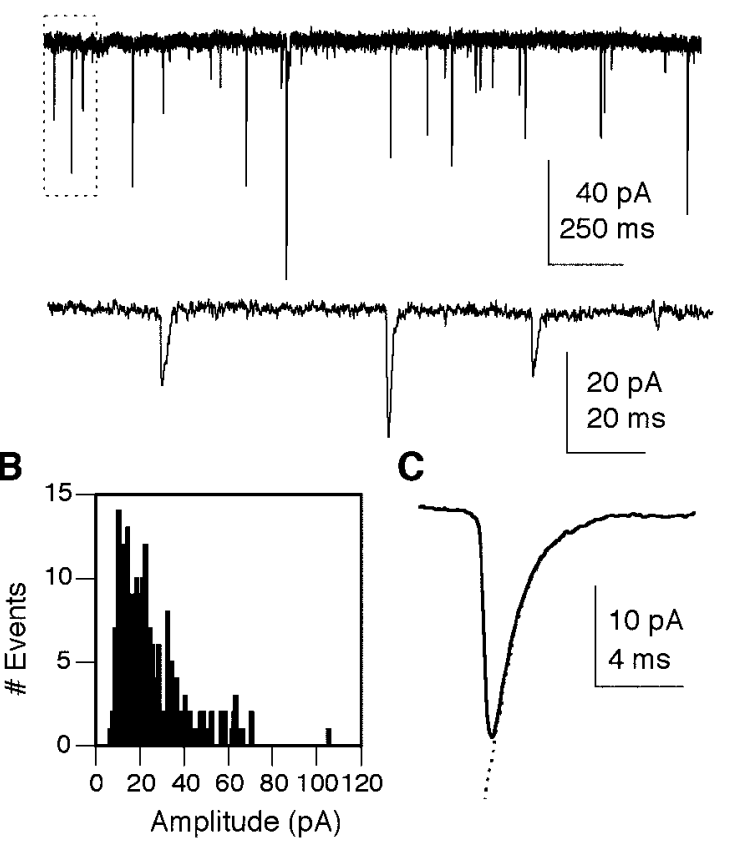

D

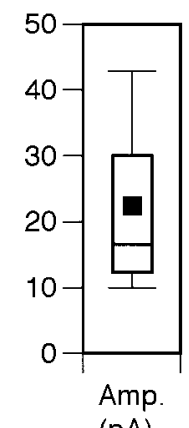

(pA)

E

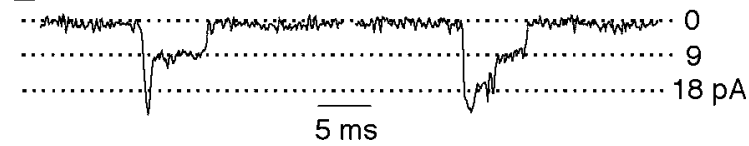

Figure 6. Biophysical properties of cholinergic mEPSCs in GFP + Kenyon cells. A, Lowresolution recording of synaptic currents from a GFP + neuron at 6 DIV in the presence of TTX. Holding potential, $-75 \mathrm{mV}$. The area indicated by the box in the upper trace is expanded in the second trace. $B$, An mEPSC amplitude histogram constructed from 223 individual events recorded in this neuron illustrates the typical positively skewed distribution. $C$, The ensemble average $\mathrm{mEPSC}$ from this same neuron exhibits a typical rapid rise and decay phase that is well fitted by a single exponential (indicated by the dotted line; $\tau=1.35 \mathrm{msec}$ ). $D$, Box plots of the mEPSC amplitude, rise time, and decay time constant obtained from analysis of $38 \mathrm{GPF}+$ Kenyon cells. Box points represent the 10th, 25 th, median, 75 th, and 95 th percentiles. Values are means \pm SEM. E, Examples of two mEPSCs ending in discrete steps characteristic of $\sim 10 \%$ of the synaptic currents observed. Holding potential, $-75 \mathrm{mV}$. Cultures were prepared from 0K107-GAL4;UAS-GFP pupae.

mEPSCs were quantified in terms of frequency and biophysical properties. Cells were included in the analysis only if a stable whole-cell recording was maintained for a minimum of 1-2 min and the mEPSC frequency was $0.1 \mathrm{~Hz}$ or greater (Fig. 6A). A typical amplitude histogram, generated from a single cell reveals a positively skewed distribution (Fig. 6B). An ensemble average current from the same cell illustrates the characteristic rapid rate of rise and a decay phase that is well fitted by a single exponential $(\tau=1.35 \mathrm{msec}$ ) (Fig. 6C). The amplitude, rise time, and decay
Table 1. Comparison of synaptic currents in GFP + Kenyon cells and GFP - neurons in 0K107-GAL4;UAS-GFP cultures

\begin{tabular}{lccll}
\hline & GFP+ Kenyon cells & GFP - neurons & GFP+ Kenyon cells & GFP - neurons \\
& $\mathrm{mEPSC}(n=38)$ & $\mathrm{mEPSC}(n=35)$ & $\mathrm{mIPSC}(n=12)$ & $\operatorname{mIPSC}(n=30)$ \\
\hline Frequency $(\mathrm{Hz})$ & $1.2 \pm 0.4$ & $1.0 \pm 0.32$ & $0.89 \pm 0.25$ & $1.10 \pm 0.3$ \\
Amplitude (pA) & $22.1 \pm 2.1$ & $25.4 \pm 3.9$ & $24.6 \pm 3.8$ & $25.4 \pm 4.4$ \\
Rise time (msec) & $0.43 \pm 0.05$ & $0.45 \pm 0.06$ & $0.80 \pm 0.20$ & $0.69 \pm 0.11$ \\
Decay $\tau(\mathrm{msec})$ & $1.4 \pm 0.1$ & $1.5 \pm 0.1$ & $3.7 \pm 0.9$ & $3.3 \pm 0.5$ \\
\hline
\end{tabular}

All cultures were prepared from OK107-GAL4:UAS-GFP pupae. Neurons were identified as GFP + or GFP - with fluorescent illumination just before electrophysiological characterization. No significant differences were noted between GFP + Kenyon cells and GFP - neurons ( $p>0.05$; Student's $t$ test; unpaired).

time constant were determined from ensemble average currents, and the box plots indicate the mean and variability determined from 38 GFP + Kenyon cells (Fig. 6D, Table 1). Although there was considerable heterogeneity within this population, none of these parameters varied significantly as a function of age in culture (data not shown).

Previous studies, at the Drosophila neuromuscular junction, have reported one or more discrete steps on the falling phase of some of the miniature end-plate currents (Kidokoro and Nishikawa, 1994). These steps are thought to represent the activity of synaptically localized glutamate channels (Nishikawa and Kidokoro, 1995). Although all of the individual EPSCs in the cultured neurons used for biophysical properties described above were characterized by a single exponential decay phase, $\sim 10 \%$ of the mEPSCs in most cells ended in a discrete step (or steps) (Fig. 6E). The conductance, calculated from the mean step amplitude measured from 12 or more individual synaptic events in each of five neurons was $121 \pm 15 \mathrm{pS}$. These step currents were eliminated in the presence of curare, indicating that they are likely to represent activity of single nAChR channels localized at central synapses.

\section{GABAergic miniature IPSCs in GFP+ Kenyon cells}

To examine GABAergic miniature inhibitory postsynaptic currents (mIPSCs) in isolation, recordings were made in the presence of TTX to block voltage-gated sodium channels and at $0 \mathrm{mV}$, the reversal potential for cholinergic EPSCs. GABAergic mIPSCs were outward at $0 \mathrm{mV}$ and were completely and reversibly blocked by $2 \mu \mathrm{M}$ PTX $(n=4)$, a classic insect GABA receptor antagonist (Fig. 7A). They were not blocked by the vertebrate $\mathrm{GABA}_{\mathrm{A}}$ receptor antagonist BMC $(2-100 \mu \mathrm{M}, n=4)($ Fig. $7 A)$. The mIPSCs were quantified in terms of frequency and biophysical properties. Cells were included in the analysis only if a stable whole-cell recording was maintained for a minimum of 1-2 min and the mIPSC frequency was $0.1 \mathrm{~Hz}$ or greater (Fig. $7 B$ ). An amplitude histogram generated from a single cell reveals a positively skewed distribution (Fig. 7C). An ensemble average current from the same cell illustrates the characteristic rapid rate of rise and a decay phase that is well fitted by a single exponential $(\tau=$ $2.67 \mathrm{msec}$ ) (Fig. 7C). The amplitude, rise time, and decay time were determined from ensemble average currents, and the box plots indicate the mean and variability of these parameters observed in 12 GFP + Kenyon cells (Fig. 7D, Table 1). Both the amplitude and decay showed a wide range in the Kenyon cell population, but neither varied consistently with age in culture.

\section{Expression of GFP does not alter properties of cholinergic or GABAergic currents}

To determine whether the $\mathrm{nAChRs}$ or GABA receptors mediating synaptic transmission in GFP + Kenyon cells were different from the GFP-negative $(\mathrm{GFP}-)$ cell population, we compared the properties of both the mEPSCs and mIPSCs in these two groups 
A

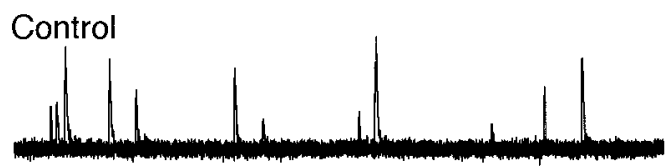

$\mathrm{BMC}$

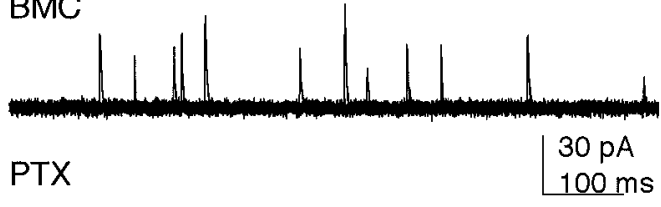

Wash

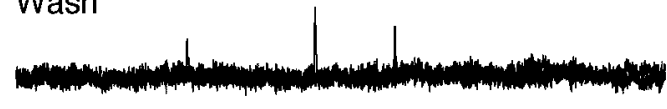

B

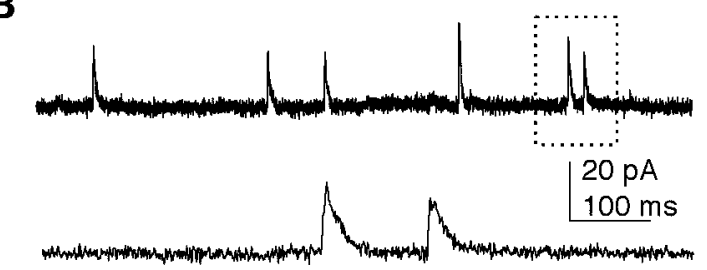

C
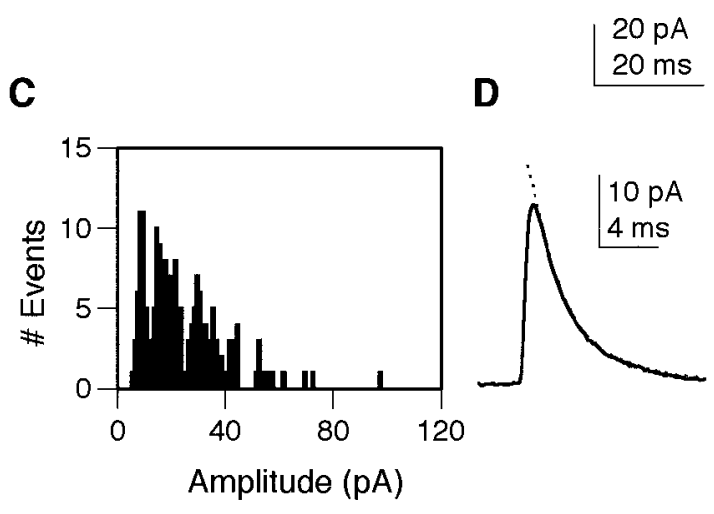

$\mathbf{E}$

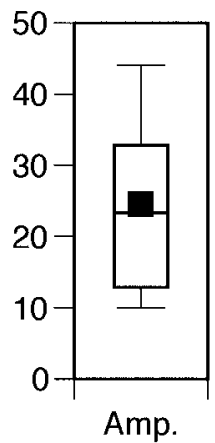

(pA)

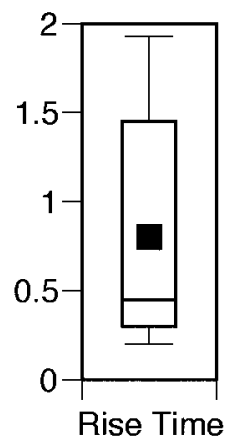

(ms)

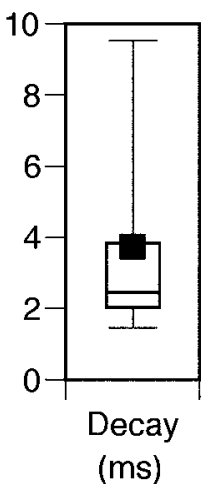

(ms)
Figure 7. Pharmacological and biophysical properties of GABAergic synaptic currents in GFP+ Kenyon cells. A, PTX $(2 \mu \mathrm{M})$ reversibly blocks the fast outward currents recorded at $0 \mathrm{mV}$ in the presence of curare. These currents are not blocked by BMC $(2 \mu \mathrm{M})$. Superimposed current traces (three sweeps) from a single neuron at $4 \mathrm{DIV}$, at a holding potential of $0 \mathrm{mV}$, during bath perfusion of the indicated drugs. $B$, Low-resolution recording of outward currents from a neuron at 5 DIV at a holding potential of $0 \mathrm{mV}$ in the presence of TTX. The area indicated in the box is expanded in the second trace. C, An mIPSC amplitude histogram constructed from 184 individual events recorded in this neuron illustrates the positively skewed distribution. D, The ensemble average mIPSC from this same neuron exhibits a rapid rise and decay phase that is well fitted
Table 2. mEPSC properties in GFP + cholinergic neurons in Cha-GAL4 cultures and in wild-type neurons examined in the absence of fluorescent illumination in Canton-S cultures

\begin{tabular}{llc}
\hline & $\begin{array}{l}\text { GFP }+ \text { cholinergic neurons } \\
\text { Cha-Gal4 mEPSCs } \\
(n=11)\end{array}$ & $\begin{array}{l}\text { Wild-type neurons } \\
\text { Canton-S mEPSCs } \\
(n=10)\end{array}$ \\
\hline Frequency (Hz) & $0.66 \pm 0.12$ & $0.68 \pm 0.19$ \\
Amplitude (pA) & $27.5 \pm 2.8$ & $24.5 \pm 2.7$ \\
Rise time (msec) & $0.39 \pm 0.07$ & $0.3 \pm 0.02$ \\
Decay $\tau$ (msec) & $1.7 \pm 0.21$ & $1.5 \pm 0.12$ \\
\hline
\end{tabular}

Recordings were obtained from GFP+ cholinergic neurons in cultures prepared from Cha-GAL4 pupae and in neurons examined in the absence of fluorescent illumination in wild-type cultures prepared from Canton- $S$ pupae. There were no significant differences in the mEPS ( properties between these two groups or between either of these groups and the GFP + Kenyon cells in OK107-GAL4;UAS-GFP cultures (Table 1).

(Table 1). No significant differences were detected when the values in GFP + and GFP - neurons were compared ( $p>0.05$, Student's $t$ test, unpaired). This suggests that although there is considerable variability in receptor properties observed among individual neurons, the Kenyon cells do not express receptors that distinguish these neurons from the general population. However, it is formally possible that the expression of GFP actually masked a difference between the Kenyon cell and nonKenyon cell groups. Therefore, synaptic currents were examined in GFP+ neurons in cultures made from the Cha-GAL4 line. In these cultures, the GFP + neurons represent a cholinergic population. There were no differences in the properties of the mEPSCs in GFP+ cholinergic neurons in Cha-GAL4 cultures (Table 2) and GFP+ Kenyon cells in OK107-GAL4;UAS-GFP cultures (Table 1). Finally, receptor properties were not influenced by fluorescent illumination before recording, based on similarities in the synaptic currents in neurons examined in wild-type Canton-S cultures in the absence of fluorescent illumination (Table 2).

\section{Discussion}

The technical challenge of recording from single neurons has limited functional studies of synaptic transmission in the adult Drosophila CNS. An alternative approach, examination of the functional properties of synapses formed between neurons in cell culture, has demonstrated that fast synaptic transmission is mediated by nAChRs and GABA receptors in embryonic Drosophila neurons (Lee and O'Dowd, 1999; Kuppers et al., 2003; Lee et al., 2003; Rohrbough et al., 2003). It has also been feasible to record from identified Drosophila mushroom body Kenyon cells, in cultures prepared from third instar larvae (Wright and Zhong, 1995; Delgado et al., 1998). However, although the larval Kenyon cells expressed voltage-gated potassium channels, there was no evidence of synaptic currents. Here we describe a culture system that supports the formation of synaptic connections between neurons harvested from late-stage pupal brains, enabling description of receptors that mediate fast synaptic transmission in neurons that form the adult Drosophila CNS.

To identify the properties of receptors mediating synaptic currents specifically in the mushroom body Kenyon cells, cultures were prepared from OK107-GAL4;UAS-GFP pupae. The GFP+ neurons in culture are likely to represent Kenyon cells for the following reasons. First, the majority of GFP + neurons in the

$\leftarrow$

by a single exponential (indicated by the dotted line; $\tau=2.67 \mathrm{msec}$ ). $E$, Box plots of the $\mathrm{mEPSC}$ amplitude, $10-90 \%$ rise time, and decay time constant obtained from the analysis of $12 \mathrm{GFP}+$ Kenyon cells. Box points represent the 10th, 25th, median, 75th, and 95th percentiles. Values are means \pm SEM. Cultures were prepared from 0K107-GAL4;UAS-GFP pupae. 
central brain regions of these pupae were located in the dorsal posterior region of the brain with an axonal projection pattern and small cell body size characteristic of Kenyon cells (Lee et al., 1999). In culture, there was a wide range in cell body size in the general population of neurons, but the majority of GFP + neurons had small soma diameters. In addition, the whole-cell capacitance, which is proportional to membrane surface area, was significantly smaller in GFP + versus GFP - neurons. Second, there are $\sim 5000$ Kenyon cells per brain (Ito et al., 1998). In cultures prepared from single brains, the maximal number of GFP + neurons was 2400, with a mean of 1300 , representing a recovery rate of $\sim 25 \%$.

Electrophysiological analysis demonstrates that nAChRs mediate the vast majority of spontaneously occurring fast excitatory synaptic currents in the cultured Kenyon cells. A functional study in a honeybee whole-brain preparation reported that the population spike in field potential recordings from the mushroom body region, evoked by stimulating the antennal lobe, was dramatically reduced by curare and $\alpha$-BTX (Oleskevich, 1999). In contrast, the population spike amplitude was not significantly altered by the NMDA and AMPA/kainate glutamate receptor blockers APV and CNQX. These data support the hypothesis that nAChRs mediate fast excitatory synaptic transmission in Kenyon cells in vivo, as they do in vitro. However, we cannot rule out the possibility that other receptors subtypes found in the Drosophila CNS are also involved in fast excitatory synaptic transmission in Kenyon cells in vivo. Northern blot analysis demonstrates that at least four ionotropic glutamate receptors, DGluR-1A,B and DNMDAR-I and II, are expressed in the adult Drosophila CNS (Ultsch et al., 1992, 1993; Volkner et al., 2000). Antibody staining suggests that DGluR1-B is not likely to be directly involved in mediating synaptic transmission in Kenyon cells because this protein is expressed primarily in lateral neurons, a small subset of cells in the central brain region (Volkner et al., 2000). The potential role of the other three glutamate receptor subtypes cannot be similarly evaluated because we are not aware of any reports describing the cellular localization of these proteins in the adult CNS.

The rapid rise and decay kinetics of the nAChR-mediated synaptic currents is not limited to the GFP+ Kenyon cells, but it is characteristic of the general population of neurons. This suggests that the basic properties of receptors mediating fast excitatory synaptic transmission can be similar, even in distinct populations of neurons, distinguished by the neurotransmitter phenotype or anatomical grouping. This is not entirely unexpected, given that the kinetic properties of AMPA-receptor-mediated mEPSCs in the mammalian CNS can be similar in anatomically and functionally distinct neuronal subpopulations (Gardner et al., 1999; Simkus and Stricker, 2002). More detailed analysis of receptor properties in distinct cell types, identified using enhancer trap lines defining specific neuronal subtypes, may reveal differences in functional properties not detected in the present study.

The excitatory nAChR-mediated currents in Kenyon cells are also $\alpha$-BTX-sensitive. Although the subunit composition of native nAChRs in Drosophila is not known, these data suggest that they may include ALS or D $\alpha 3$, subunits that confer $\alpha$-BTX sensitivity to receptors expressed in Xenopus oocytes (Bertrand et al., 1994; Schulz et al., 1998). In mammalian systems, $\alpha$-BTX sensitive neuronal nAChRs have a high calcium permeability (Bertrand et al., 1993; Seguela et al., 1993). Preliminary calcium imaging studies in the Drosophila cultures demonstrate that activation of nAChRs in GFP+ Kenyon cells causes a rapid increase in intracellular calcium levels even in the presence of cobalt, a voltage-gated calcium channel blocker (Campusano and O'Dowd, unpublished observations). Therefore, in addition to mediating rapid depolarization of the membrane, activation of the receptors could play a role in the calcium-dependent regulation of gene expression.

Reversible blockade of neurotransmitter release from Drosophila mushroom body Kenyon cells, during different phases of training in an olfactory associative learning paradigm, has demonstrated that synaptic transmission between Kenyon cells and their downstream partners is important in memory retrieval but is not necessary for memory acquisition or storage (Dubnau et al., 2001). This suggests that synaptic receptors in mushroom body Kenyon cell dendrites are sites of functional plasticity important in the acquisition and storage of memory. Repetitive activation of cholinergic afferents during olfactory associative conditioning could result in long-lasting modifications in synaptic transmission at cholinergic synapses in Kenyon cells through at least two different mechanisms. First, if the activation of nAChRs results in an increase in intracellular calcium, repeated stimulation may be sufficient to trigger changes in gene expression that could affect the number and/or type of synaptic receptors expressed or could alter the interaction of Kenyon cells with their postsynaptic partners. Second, it is possible that heterosynaptic facilitation contributes to plasticity at these synapses. Dopamine and octopamine receptors are expressed at high levels in the mushroom body region, and activation of these receptors can regulate cAMP levels in S2 cells (Han et al., 1996, 1998). In addition, repetitive spaced db-cAMP exposure can cause a persistent increase in transmission at cholinergic synapses in cultured embryonic neurons (Lee and O'Dowd, 2000). Therefore, if the unconditioned stimulus during olfactory conditioning activates interneurons synapsing on cholinergic terminals in the mushroom bodies, corelease of biogenic amines could modulate cAMP levels, resulting in a persistent increase in transmission at these synapses.

In addition to receiving excitatory cholinergic input, the GFP + Kenyon cells also express GABA receptors that mediate fast inhibitory synaptic transmission. Similar to the GABA receptors in the general population of neurons, these are PTX-sensitive and are not blocked by BMC. Based on localization studies (Harrison et al., 1996) and their pharmacological profile, it is tempting to speculate that these synaptic receptors contain Rdl subunits, similar to the PTX-sensitive synaptic GABA receptors described previously in embryonic cultured neurons (Lee et al., 2003). However, there are some significant differences in the GABAergic synaptic currents in the pupal and embryonic neurons. First, the mean mIPSC amplitude is 2-3 times larger in GFP + Kenyon cells and pupal neurons in general, compared with embryonic neurons. Second, the mean mIPSC decay in the pupal neurons is faster than that seen in the embryonic neurons, and the agedependent change in this property noted in embryonic neurons is not seen at the pupal stage. These differences could arise from the stage-specific regulation of receptor density, quantal size, and/or subunit composition. It should be noted that there is a broad range in the decay time constants observed even within pupal neurons, suggesting that GABA receptor expression may be regulated in a cell-specific manner.

GABA receptors mediating fast inhibitory input in cultured Kenyon cells is consistent with studies demonstrating GABApositive synaptic terminals on Kenyon cells in vivo (Yusuyama et al., 2002). In locust, some of the GABAergic inputs to the mushroom bodies originate from neurons in the lateral horn, a structure that receives excitatory input from cholinergic antennal lobe projection neurons in response to olfactory stimuli (Perez-Orive et al., 2002). Therefore, the presentation of olfactory stimuli during olfactory associative learning should activate GABAergic, as 
well as cholinergic, afferents to the Drosophila mushroom bodies. This suggests that activity-dependent alterations in transmission at the GABAergic synapses, like cholinergic synapses, could also be important in mediating long-lasting modifications contributing to learning and memory in Drosophila.

In summary, these studies demonstrate that nAChRs and GABA receptors mediate fast synaptic transmission in Kenyon cells; therefore, they are in a position to be activated during olfactory associative learning. Preliminary data indicate that both dopamine and octopamine can modulate transmission at cholinergic and GABAergic synapses in the cultured pupal neurons, suggesting that heterosynaptic facilitation may be involved in regulating plasticity at these synapses ( $\mathrm{Su}$ and O'Dowd, unpublished observations). The ability to examine regulation of synaptic currents in identified populations of cells important in mediating olfactory learning and memory, in combination with access to mutants that alter specific aspects of this complex behavior, provide a unique set of tools for exploring the relationship between synaptic plasticity and learning.

\section{References}

Bainbridge S, Brownes M (1981) Staging the metamorphosis of Drosophila melanogaster. J Embryol Exp Morphol 66:57-80.

Bertrand D, Galzi JL, Devillers-Thiery A, Bertrand S, Changeux EX (1993) Mutations at two distinct sites within the channel domain M2 alter calcium permeability of neuronal $\alpha 7$ nicotine receptors. Proc Natl Acad Sci USA 90:6971-6975.

Bertrand D, Ballivet M, Gomez M, Bertrand S, Phannavong B, Gundelfinger ED (1994) Physiological properties of neuronal nicotinic receptors reconstituted from the vertebrate B2 subunit and Drosophila alpha subunits. Eur J Neurosci 6:869-875.

Bicker G (1999) Histochemistry of classical neurotransmitters in antennal lobes and mushroom bodies of the honeybee. Microsc Res Tech 45:174-183.

Cayre M, Buckingham SD, Yagodin S, Sattelle DB (1999) Cultured insect mushroom body neurons express functional receptors for acetylcholine, GABA, glutamate, octopamine, and dopamine. J Neurophysiol 81:1-14.

Connolly JB, Roberts IJH, Armstrong D, Kaiser K, Forte M, Tully T, O'Kane CJ (1996) Associative learning disrupted in impaired Gs signaling in Drosophila mushroom bodies. Science 274:2104-2107.

Deglise P, Grunewald B, Gauthier M (2002) The insecticide imidacloprid is a partial agonist of the nicotinic receptor of honeybee Kenyon cells. Neurosci Lett 321:13-16.

Delgado R, Davis R, Bono MR, Latorre R, Labarca P (1998) Outward currents in Drosophila larval neurons: dunce lacks a maintained outward current component downregulated by cAMP. J Neurosci 18:1399-1407.

Dubnau J, Grady L, Kitamoto T, Tully T (2001) Disruption of neurotransmission in Drosophila mushroom body blocks retrieval but not acquisition of memory. Nature 411:476-480.

Ganeshina O, Menzel R (2001) GABA-immunoreactive neurons in the mushroom bodies of the honeybee: an electron microscopic study. J Comp Neurol 437:335-349.

Gardner SM, Trussell LO, Oertel D (1999) Time course and permeation of synaptic AMPA receptors in cochlear nuclear neurons correlate with input. J Neurosci 19:8721-8729.

Goldberg F, Grunewald B, Rosenboom H, Menzel R (1999) Nicotinic acetylcholine currents of cultured Kenyon cells from the mushroom bodies of the honeybee Aapis mellifera. J Physiol 514 (Pt 3):759-768.

Han K-A, Millar NS, Davis RL (1998) A novel octopamine receptor with preferential expression in Drosophila mushroom bodies. J Neurosci 18:3650-3658.

Han K-A, Millar NS, Grotewiel MS, Davis RL (1996) DAMB, a novel dopamine receptor expressed specifically in Drosophila mushroom bodies. Neuron 16:1127-1135.

Hansson B (2002) A bug's smell: research into insect olfaction. Trends Neurosci 25:270-274

Harrison JB, Chen HH, Sattelle E, Barker PJ, Huskisson NS, Rauh JJ, Bai D, Sattelle DB (1996) Immunocytochemical mapping of a C-terminus anti-peptide antibody to the GABA receptor subunit, RDL in the nervous system of Drosophila melanogaster. Cell Tissue Res 284:269-278.
Heisenberg M (1998) What do the mushroom bodies do for the insect brain: an introduction. Learn Mem 5:1-10.

Hilgenberg LG, Hoover CL, Smith MA (1999) Evidence of an agrin receptor in cortical neurons. J Neurosci 19:7384-7393.

Ito K, Suzuki K, Estes P, Ramaswami M, Yamamoto D, Strausfeld NJ (1998) The organization of extrinsic neurons and their implications in the functional roles of the mushroom bodies in Drosophila melanogaster Meigen. Learn Mem 5:52-77.

Kidokoro Y, Nishikawa K (1994) Miniature endplate currents at the newly formed neuromuscular junction in Drosophila embryos and larvae. Neurosci Res 19:143-154.

Kraft R, Levine RB, Restifo LL (1998) The steroid hormone 20hydroxyecdysone enhances neurite growth of Drosophila mushroom body neurons isolated during metamorphosis. J Neurosci 18:8886-8899.

Kreissl S, Bicker G (1989) Histochemistry of acetylcholinesterase and immunocytochemistry of an acetylcholine receptor-like antigen in the brain of the honeybee. J Comp Neurol 286:71-84.

Kuppers B, Sanchez-Soriano N, Letzkus J, Technau GM, Prokop A (2003) In developing Drosophila neurones the production of gamma-amino butyric acid is tightly regulated downstream of glutamate decarboxylase translation and can be influenced by calcium. J Neurochem 84:939-951.

Lee D, O’Dowd DK (1999) Fast excitatory synaptic transmission mediated by nicotinic acetylcholine receptors in Drosophila neurons. J Neurosci 19:5311-5321.

Lee D, O'Dowd DK (2000) cAMP-dependent plasticity at excitatory cholinergic synapses in Drosophila neurons: alterations in the memory mutan dunce. J Neurosci 20:2104-2111.

Lee D, Su H, O'Dowd DK (2003) GABA receptors containing Rdl subunits mediate fast inhibitory synaptic transmission in Drosophila neurons. J Neurosci 23:4625-4634.

Lee T, Lee A, Luo L (1999) Development of the Drosophila mushroom bodies: sequential generation of three distinct types of neurons from a neuroblast. Development 126:4065-4076.

Nishikawa K, Kidokoro Y (1995) Junctional and extrajunctional glutamate receptor channels in Drosophila embryos and larvae. J Neurosci 15:7905-7915.

Oleskevich S (1999) Cholinergic synaptic transmission in insect mushroom bodies in vitro. J Neurophysiol 82:1091-1096.

Perez-Orive J, Mazor O, Turner GC, Cassenaer S, Wilson RI, Laurent G (2002) Oscillations and sparsening of odor representations in the mushroom body. Science 297:359-365.

Rohrbough J, O’Dowd DK, Baines RA, Broadie K (2003) Cellular bases of behavioral plasticity: establishing and modifying synaptic circuits in the Drosophila genetic system. J Neurobiol 54:254-271.

Salvaterra PM, Kitamoto T (2001) Drosophila cholinergic neurons and processes visualized with Gal4/UAS-GFP. Gene Exp Patterns 1:73-82.

Schulz R, Sawruk E, Mulhardt C, Bertrand S, Baumann A, Phannavong B, Betz H, Bertrand D, Gundelfinger ED, Schmitt B (1998) D $\alpha 3$, a new functional $\alpha$ subunit of nicotinic acetylcholine receptors from Drosophila. J Neurochem 71:853-862.

Seguela P, Wadiche J, Dineley-Miller K, Dani JA, Patrick JW (1993) Molecular cloning, functional properties, and distribution of $\alpha 7$ : a nicotinic cation channel highly permeable to calcium. J Neurosci 13:596-604.

Simkus CR, Stricker C (2002) Properties of mEPSCs recorded in layer II neurones of rat barrel cortex. J Physiol (Lond) 545:509-520.

Theunissen F (2003) From synchrony to sparseness. Trends Neurosci 26:61-64.

Ultsch A, Schuster CM, Laube B, Betz H, Schmitt B (1993) Glutamate receptors of Drosophila melanogaster: primary structure of a putative NMDA receptor protein expressed in the head of the adult fly. FEBS Lett 324:171-177.

Ultsch A, Schuster CM, Laube B, Schloss P, Schmitt B, Betz H (1992) Glutamate receptors of Drosophila melanogaster: cloning of a kainateselective subunit expressed in the central nervous system. Proc Natl Acad Sci USA 89:10484-10488.

Volkner M, Lenz-Bohme B, Betz H, Schmitt B (2000) Novel CNS glutamate receptor subunit genes of Drosophila melanogaster. J Neurochem 75:1791-1799.

Wright ND, Zhong Y (1995) Characterization of $\mathrm{K}^{+}$currents and the cAMP-dependent modulation in cultured Drosophila mushroom body neurons identified by lacZ expression. J Neurosci 15:1025.

Yusuyama K, Meinertzhagen IA, Schurmann FW (2002) Synaptic organization of the mushroom body calyx in Drosophila melanogaster. J Comp Neurol 445:211-226. 